\title{
Remarks on the Idea of Non-monotonic (Diagrammatic) Inference
}

\author{
Observaciones sobre la idea de inferencia no monotónica \\ (diagramática) \\ J. Martín Castro-Manzano \\ Universidad Popular del Estado de Puebla, México \\ josemartin.castro@upaep.mx \\ Recibido: 18/06/2016 • Aceptado: 02/02/2017
}

\begin{abstract}
Resumen

After a brief review of the notion of dia-

Luego de una breve revisión de la noción grammatic inference, we show in which de inferencia diagramática, mostramos sense VENN provides a logical framework able to model some form of nonmonotonic diagrammatic inference. This result suggests that, just as there are classical and non-classical sentential logical systems, there may be classical and nonclassical diagrammatic logical systems. en qué sentido VENN provee un marco lógico capaz de modelar cierta forma de inferencia diagramática no-monotónica. Este resultado indica que, así como existen sistemas lógicos sentenciales clásicos y no-clásicos, pueden existir sistemas lógicos diagramáticos clásicos y no-clásicos.

Keywords: Classical logic, diagrammatic reasoning, nonclassical logic, VENN diagrams.

Palabras clave: Lógica clásica, razonamiento diagramático, lógica no-clásica, diagramas de VEEN.
\end{abstract}


"Diagrammatic reasoning is the only really fertile reasoning. If logicians would only embrace this method, we should no longer see attempts to base their science on the fragile foundations of metaphysics or a psychology not based on logical theory; and there would soon be such an advance in logic that every science would feel the benefit of it"

Peirce, 1906

\section{Introduction}

While the representational advantages of diagrams have been widely recognized, their computational and logical virtues have not enjoyed the same recognition up until recently. Notable examples in the history of Logic and Science suggest a recognition of these representational features $;^{1}$ but, despite this acknowledgement, when it comes to the computational and logical nature of reasoning, there is still a tradition that supports the claim that, while proof-based reasoning is essential in logic and mathematics, diagram-based reasoning, no

1 Notable examples include the diagrams of the square of opposition, usually attributed to Apuleius (Londey and Johanson, 1987: 109), and the diagrams for syllogisms imputed to Ammonius Hermiae or Philoponus (Hamilton, Mansel and Veitch, 1865: 420). Llull's Ars Magna is exemplar in that it included a diagrammatic device used to explain divine nature to those unable to understand God, as if diagrammatic methods were more convincing or expressive than sentential representations (Llull, 1501). Thomas Murner used diagrams in his Logica Memorativa in order to teach logic (Murner, 1509). Dutch mathematician Simon Stevin developed another remarkable diagram in his demonstration that the efficiency of the inclined plane is a logical consequence of the impossibility of perpetual motion (Stevin, 1586). And, of course, we have the case of Descartes who, along with other Renaissance thinkers, like Kepler (Kepler, Aiton, Duncan, and Field, 1997), made good use of diagrams in order to model hypotheses, such as the mechanics of the pineal gland (Descartes, Miller and Miller, 1984). Even Kant, together with Jäsche, seems to have developed some sort of logic diagrams in the Jäsche Logik (Kant and Young, 1992) in order to explain the general theory of logic. 
matter how useful (Nelsen, 1993) or elegant (Polster, 2004), is not, for it is not bona fide reasoning. ${ }^{2}$

Thus, for example, Lagrange remarked in the Preface to the first edition of his Mécanique Analytique that no figures were to be found in his work (Lagrange, Boissonnade and Vagliente, 1997). Leibniz shared a similar opinion at some point (Leibniz, Remnant and Bennett, 1996: 309). Dieudonné urged a strict adherence to axiomatic methods with no appeal to geometric intuition, at least in formal proofs (Dieudonné, 1996). Tennant once suggested a diagram is only a heuristic to prompt certain trains of inference (Tennant, 1986). And today the same belief is maintained, for example, in books and manuals that cover proof techniques (Cf. Hammack, 2013).

This bias against diagram-based reasoning is based upon the assumption that diagrams, due to their spatial features, are naturally prone to fallacies, mistakes, and are not susceptible of generalization. Nevertheless, since the works of Shin (1994), Allwein, Barwise and Etchemendy (1996), a successful research program around heterogeneous and diagrammatic reasoning has promoted different studies and model theoretic schemes that help us represent and better understand diagrammatic reasoning in logical terms (Nakatsu, 2010), thus allowing well defined conceptions of logic diagrams and diagrammatic inference. Research within this program is increasing (Moktefi and Shin, 2013) and our contribution follows its main guidelines while adding a little twist on the notion of diagrammatic inference.

The little twist we suggest here has to do with the nature of diagrammatic inference. Thus, after a brief review of the notion of diagrammatic inference, we show in what sense VENN provides a logical framework able to model some form of non-monotonic diagrammatic inference. This result suggests that, just as there are classical and non-classical sentential logical systems, so to speak, there may be classical and non-classical diagrammatic logical systems.

2 This is usually the case, despite the fact that there are several instances of logic diagrams developed explicitly to perform inference: Leibniz and Couturat, 1961; Lambert, 1764; Bök, 1766; Venn, 1881; Carroll, 1887; Peirce, 1906; Karnaugh, 1954; Englebretsen, 1992; Shin, 1994 and Pagnan, 2012. 
Logic and diagrams

\section{Logical systems}

Reasoning is a process that produces new information given previous data, by following certain norms that allow us to describe inference as the unit of measurement of reasoning: inference may be more or less (in)correct depending on the compliance or violation of such norms. ${ }^{3}$ Logical systems, the tools used to model and better understand inference, may be defined by pairs of the form $\langle L, B>$, where $L$ stands for a language, and $B$ for a semantic base (often equivalent to a calculus). Usually, some syntax is used to determine, uniquely and recursively, the well formed expressions of the system; while semantics is used to provide meaning to such expressions.

\section{Diagrams}

In order to represent knowledge, we use internal and external representations. Internal representations convey mental images, for example; while external representations include physical objects on paper, blackboards, or computer screens. External representations can be further divided into two kinds: sentential and diagrammatic (Larkin and Simon, 1987).

Sentential representations, as the name indicates, are sequences of sentences in a particular language. Diagrammatic representations, on the other hand, are sequences of diagrams that contain information

3 The structural understanding of these norms has depended, typically but not exclusively, on three equivalent approaches. The semantical, whose central concept is that of interpretation and defines our notions of satisfaction, model, and logical truth; the syntactical, whose main concept is that of deducibility and characterizes our intuitions of proof, demonstration, and theorem; and the abstract one, whose idea is that of a consequence function. These approaches are usually sentential and they define inference as the proprium of logic. In particular, the classical relation of inference follows the structural norms of reflexivity, monotonicity, and cut. Consequently, a non-monotonic logic is non-classical in the sense that it does not comply with the monotonicity clause, usually because it attempts to represent defeasible inference. The typical formalisms that study this sort of inference are also sentential and they include circumscription (McCarthy, 1980), inheritance networks (Pollock, 1995; Dung, 1995), default rules (Reiter, 1980), autoepistemic logic (Moore, 1985), preferential entailment (Shoham 1987; Kraus, Lehmann and Magidor, 1990), and modal non-monotonic logic (McDermott \& Doyle, 1980). 
stored at one particular locus, including information about relations with the adjacent loci; and diagrams are information graphics that index information by location on a plane (Larkin and Simon, 1987; Nakatsu, 2010). In particular, logic diagrams are two-dimensional geometric figures with spatial relations that are isomorphic with the structure of logical statements (Gardner, 1958: 28). The difference between diagrammatic and sentential representations is that, due to this spatial feature, the former preserve explicitly information about topological relations, while the latter do not - although they may, of course, preserve other kinds of relations.

This spatial feature provides some computational advantages: diagrams group together information avoiding large amounts of search, they automatically support a large number of perceptual inferences, and they grant the possibility of applying operational constraints (like free rides and overdetermined alternatives (Shimojima, 1996)) to allow the automation of perceptual inference (Larkin and Simon, 1987).

\section{Logic with diagrams}

To wrap all this up, if reasoning is a process that produces new information given previous data and information can be represented diagrammatically, it is not uncomfortable to suggest that diagrammatic inference is the unit of measurement of diagrammatic reasoning: diagrammatic inference would be (in)correct depending on the compliance or violation of certain norms. Let us denote the relation of diagrammatic logical consequence or diagrammatic inference by - this relation would define our intuitions around the informal notions of visual inference or visual argument and would follow, ex hypothesi, classical structural norms (reflexivity, monotonicity, and cut), while the operator would follow Shimojima's definition of a free ride as a process in which some reasoner gains information without following any step specifically designed to gain it, i.e., a process that allows the reasoner to reach automatically (and sometimes inadvertently) a diagrammatic representation of a conclusion from a diagrammatic representation of premises (Shimojima, 1996: 32). VENN, as we will see in the next section, is a diagrammatic logical system of this sort. 
Syllogistic,VENN, and Non-monotonicity

The previous account of diagrammatic inference sounds reasonable, in principle; but monotonicity does not seem to be a feasible property of diagrammatic inference due to the spatial nature of diagrams, so to speak. In order to explain this, we will see in what senseVENN can be used to represent some sort of (diagrammatic) non-monotonicity and, for that, we will require a syllogistic base and a well defined diagrammatic logical system, namely VENN.

\section{Syllogistic}

Syllogistic is a term logic that has its origins in Aristotle's Prior Analytics and is the theory of inference that deals with the consequence relation between two categorical propositions taken as premises and another categorical proposition taken as a conclusion. A categorical proposition is a proposition composed by two terms, a quantity, and a quality. The subject and the predicate of a proposition are called terms: the term-schema $\mathrm{S}$ denotes the subject term of the proposition and the term-schema $\mathrm{P}$ denotes the predicate. The quantity may be either universal (All) or particular (Some) and the quality may be either affirmative or negative. These categorical propositions are denoted by a label, either A (universal affirmative), E (universal negative), I (particular affirmative), or $\mathrm{O}$ (particular negative). A categorical syllogism, then, is a sequence of three categorical propositions ordered in such a way that two propositions are premises and the last one is a conclusion. Within the premises there is a term that appears in both premises but not in the conclusion. This particular term works as a link between the remaining terms and is known as the middle term, which we denote with the term-schema M. According to this term we can set up four figures that encode all the valid and only the valid syllogisms (Table 1 ). ${ }^{4}$

4 For sake of brevity, but without loss of generality, we omit the syllogisms that require existential presupposition. 


\begin{tabular}{cccc}
\hline Figure 1 & Figure 2 & Figure 3 & Figure 4 \\
\hline Barbara & Cesare & Disamis & Calemes \\
MAPSAM $\therefore$ SAP & PAMSAM $\therefore$ SAP & MIPMAS $\therefore$ SIP & PAMMES $\therefore$ SEP \\
\hline Celarent & Camestres & Datisi & Dimaris \\
MEPSAM $\therefore$ SEP & PAMSEM $\therefore$ SEP & MAPMIS $\therefore$ SIP & PIMMAS $\therefore$ SIP \\
\hline Darii & Festino & Bocardo & Fresison \\
MAPSIM $\therefore$ SIP & PEMSIM $\therefore$ SOP & MOPMAS $\therefore$ SOP & PEMMIS $\therefore$ SOP \\
\hline Ferio & Baroco & Ferison & \\
MEPSIM $\therefore$ SOP & PAMSOM $\therefore$ SOP & MEPMIS $\therefore$ SOP & \\
\hline
\end{tabular}

Table 1. Valid syllogisms

\section{VENN}

VENN is a sound and complete diagrammatic logical system (Shin, 1994) that is equivalent to monadic First Order Logic (Hammer, 1995) and thus represents syllogistic quite perspicuously. Following (Shin, 1994: 48), it has a well defined vocabulary, syntax, and semantics. Briefly, the vocabulary is defined by the next elements: the closed curve, the rectangle, the shading, the X, and the line (Fig. 1).

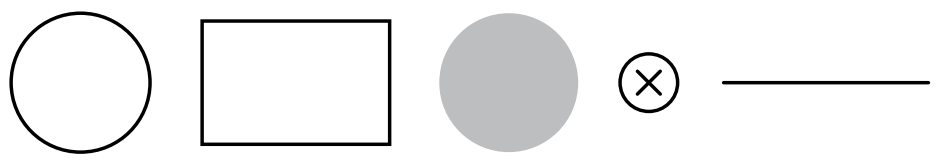

Figure 1. Syntactic elements of VENN

The semantics of VENN depends on a homomorphism with sets that helps define a diagram as any finite combination of diagrammatic elements where a region is any enclosed area in a diagram. A basic region is a region enclosed by a rectangle or a closed curve. A minimal region is a region within which no other region is enclosed. An $X$ sequence is a diagram of alternating $X$ 's and lines with an $X$ in each extremal position. Regions represent sets and the rectangle represents the domain. A shaded region represents an empty region and a region 
with an $X$ represents a non-empty region. With these definitions, a syntax for the categorical propositions can be elaborated (Fig. 2).
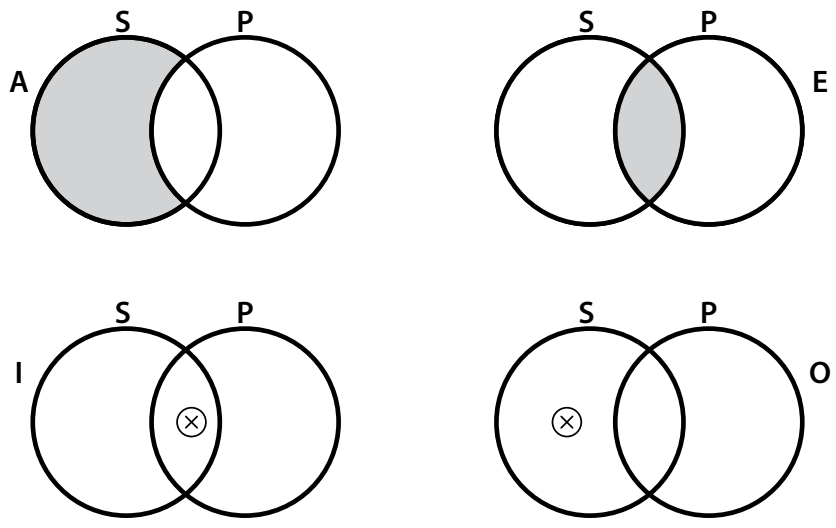

Figure 2. Categorical propositions in VENN

The rules for this diagrammatic system are six (Shin, 1994: 8193). (I) The rule of erasure of a diagrammatic object tells us that a well formed diagram (wfd) $D_{1}$ is obtained from a wfd $D$ if $D_{1}$ results from either erasing a closed curve of $D$, or erasing a shading of some region of $D$, or erasing an entire $\mathrm{X}$-sequence of $D$. (II) The rule of erasure of parts of an $X$-sequence says that $D_{1}$ is obtained from $D$ if it results from $D$ by the erasure of parts in some $\mathrm{X}$-sequence that fall in shaded regions, provided that the remaining X's are reconnected. (III) The rule of spreading of an $X$-sequence tells us that $D_{1}$ is obtained from $D$ if extra $X$-sequences have been added to some $X$-sequence of D. (IV) The rule of introduction of a basic region indicates that a basic region may be introduced by drawing either a rectangle or a closed curve. (V) The rule of conflicting information says that if a diagram has a region with both a shading and an X-sequence, then we may transform the given diagram into any diagram. Finally, (VI) the rule of unification of diagrams says that $D_{1}$ is obtained from two well formed diagrams $D_{2}$ and $D_{3}$ if every region of $D_{1}$ is a counterpart region of either $D_{2}$ or $D_{3}$ and conversely. If any region of $D$ is shaded or has an 
$\mathrm{X}$-sequence, then it has a counterpart in either $D_{1}$ or $D_{2}$ which is also shaded or has an X-sequence and conversely. ${ }^{5}$

With these rules, VENN provides an essential feature of a well defined diagrammatic logic: a diagrammatic method of decision (in this case, for syllogistic) that consists in drawing down the diagrams for the premises and then checking (by mere observation) whether it is possible to "read off" the conclusion from the drawing of the premises; in case it does, the syllogism is valid (i.e., there is a free ride); otherwise it is invalid (i.e., there is an overdetermined alternative).

\section{Example 1}

Consider a syllogism of the form MAPSIM.SSIP. According to these rules, we begin with an introduction of areas (step 1) and then a unification is applied (step 2). After that, we apply an erasure of an X-sequence (step 3) and then a spreading of an X-sequence (step 4). Finally, by the erasure of a closed curve rule, we obtain a final diagram (step 5). And since the conclusion got drawn by drawing down the premises, the diagrammatic inference is valid. This is a fair example of a free ride: MAPSIM-SIP (Fig. 3).

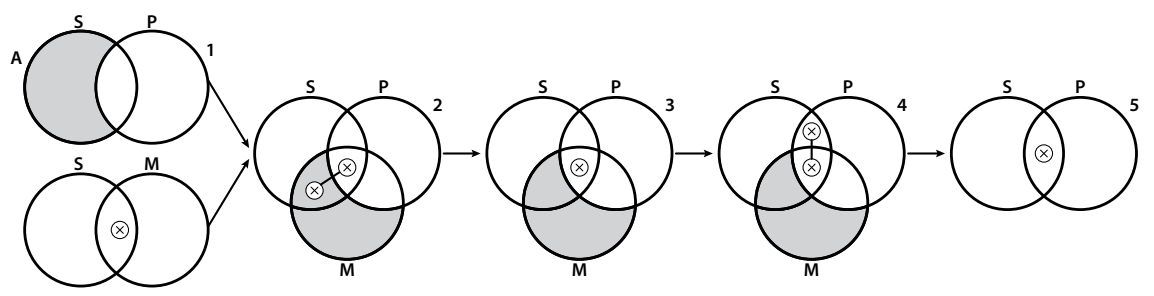

Figure 3. An example of a free ride in VENN

5 These rules may be summarized into categories of erasure, addition, and unification (Nakatsu, 2010: 133). Erasure: i) of a shading in a region; ii) of a whole X-sequence; iii) of a part of an X-sequence in a shaded region; iv) of a circle (a partial shading in a region or an $\mathrm{X}$-sequence with more than one $\mathrm{X}$ in a region). Addition: i) by lengthening an $\mathrm{X}$ sequence; ii) of a circle; iii) by partial overlapping; iv) by inclusion of all compartments of region in an X-sequence. Unification: i) by copying circles to the unified diagram; ii) by copying corresponding shading to the unified diagrams; by iii) copying corresponding $\mathrm{X}$-sequences to the unified diagrams. 


\section{Non-monotonicity with VENN}

After this brief review of diagrammatic inference, we now suggest in what sense VENN provides a logical framework able to model some form of non-monotonicity. To explain why $V E N N$ is suitable for this task, consider that non-monotonic reasoning can be characterized by the following “equation" (Bochman, 2011):

Non-monotonic reasoning $=$ Logic + Non-monotonic semantics .

We could say, in similar lines, that:

Non-monotonic diagrammatic reasoning $=$ Logic + Diagrams + Nonmonotonic semantics,

or for our purposes, that:

Non-monotonic diagrammatic reasoning $=$ Syllogistic base + VENN + Non-monotonic semantics.

To explain in what sense we can talk about some sort of nonmonotonic semantics, let us begin with an example in classical First Order Logic.

\section{Example 2}

Consider a Barbara syllogism, i.e., a reasoning of the form MAPSAMISAP:

\section{1. $\forall \mathrm{x}(\mathrm{Mx} \rightarrow \mathrm{Px}) \quad \mathrm{MAP}$}

2. $\forall \mathrm{x}(\mathrm{Sx} \rightarrow \mathrm{Mx}) \quad \mathrm{SAM}$

$\therefore \forall \mathrm{x}(\mathrm{Sx} \rightarrow \mathrm{Px}) \quad \mathrm{SAP}$

If we add the sentence SOP (which contains contradictory information w.r.t. the conclusion), we can see that SAP is still a logical consequence from MAPSAMSOP: 

1. $\forall \mathrm{x}(\mathrm{Mx} \rightarrow \mathrm{Px}) \quad \mathrm{MAP}$
2. $\forall \mathrm{x}(\mathrm{S} x \rightarrow \mathrm{Mx}) \quad \mathrm{SAM}$
3. $\exists \mathrm{x}(\mathrm{S} \mathrm{x} \wedge \neg \mathrm{Px}) \quad \mathrm{SOP}$
$\therefore \forall \mathrm{x}(\mathrm{Sx} \rightarrow \mathrm{Px}) \quad \mathrm{SAP}$

But moreover, even if we follow the account of Venn given in (Shin, 1994: 81-93) we can see that, according to rule V, since from conflicting information we can obtain any diagram, the diagram that represents $\mathrm{SAP}$ is still a logical consequence from the diagram given in MAPSAMSOP, for such rule is the diagrammatic version of the "ex contradictio sequitur quodlibet" rule, which not only implies some sort of monotonicity (Fig. 4), but also some sort of irrelevance (Fig. 5) within VENN.

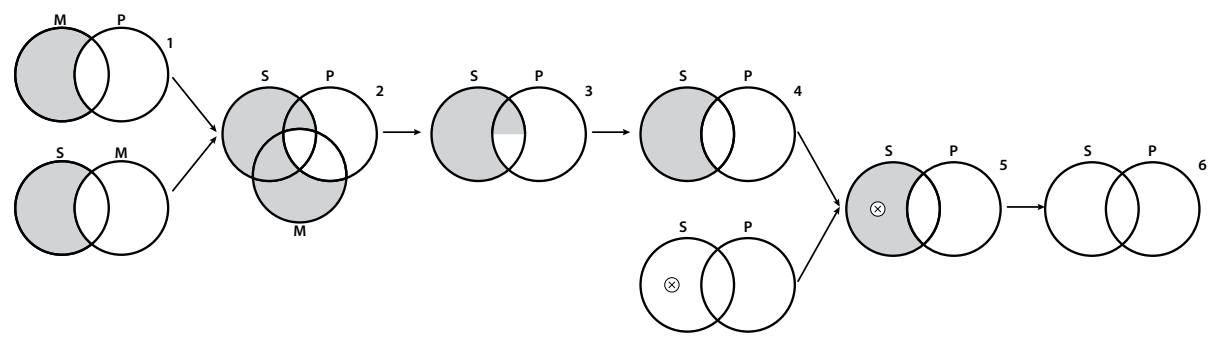

Figure 4. An example of monotonicity: MAPSAMUSOP SAP

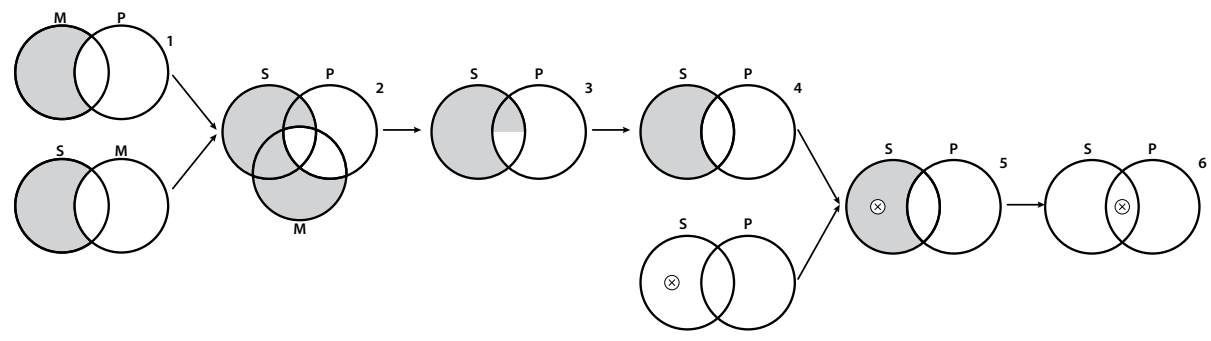

Figure 5. An example of irrelevance: MAPSAMUSOP SIP 
However, Venn diagrams are diagrams, and so, they have spatial features and this fact has consequences. Indeed, as Barwise and Etchemendy argue: "Diagrams are physical situations. They must be, since we can see them. As such they obey their own set of constraints." (Allwein et al, 1996: 23). A physical situation is a situation in the world, in this case, it is a portion of paper (i.e. a space) on which we draw the diagrams (Shimojima, 1996: 38). Hence, the constraints Barwise and Etchemendy talk about must be spatial constraints that should (dis)allow certain rules to work; in particular, the rule of conflicting information should be unlikely to work properly because contradictory diagrams cannot exist in the exact same portion of paper (i.e. the same space), because they are opposite physical situations.

To further clarify this last point, we can refer to (Shin, 1994: 167-168). Shin argues, rather convincingly, that contradictory information can be represented more perceptually in diagrammatic representations than in linguistic representations, since in diagrammatic representations this kind of information becomes visually noticeable, for conflicting spatial arrangements are evident. And to illustrate this, Shin advances a couple of examples that we are going to reproduce. For the first one, consider the following statements:

1. Susan is to the left of Tom.

2. Tom is to the left of Mary.

3. Mary is to the left of Susan.

In First Order Logic, these statements can be represented via the conjunction Lst $\wedge \mathrm{Ltm} \wedge$ Lms. This conjunction can be shown to be a contradiction by noticing that the relation "being left of" (denoted by $L x y$ ) is transitive and asymmetric. But, Shin continues, although such contradiction is not obviously revealed by the notation, it is perceptually evident with the next diagram:

Susan

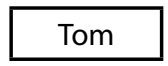

Mary

Susan 
The diagram above violates the following spatial constraint of this representation: that no diagram occupies more than one place simultaneously.

As for the second example, consider the case of a timetable, which is a diagrammatic representation of a schedule. Suppose further that we want to add a new appointment to the timetable. If this new appointment conflicts with any existing appointments, this conflict will be revealed clearly, since the new addition would violate the following spatial constraint of this representation: that no space can be occupied by more than one diagram.

What these particular examples show is that, when it comes to opposite physical situations, we must consider two physical constraints: that no diagrammatic object occupies more than one place simultaneously and that no space can be occupied by more than one diagrammatic object. And these remarks give us plausible reasons to warrant some sort of non-monotonicity within $V E N N$, provided we interpret diagrams as legitimate inference bearers with physical properties and not just as mediums that represent information. So, let us consider a couple of definitions that presuppose the general notion of a physical situation.

\section{Definition 1}

(Diagrammatic configuration) Let $\Delta=\left\{\delta_{1}, \ldots, \delta_{n}\right\}$ be a sequence of diagrams for categorical propositions in VENN, let StP be any categorical proposition in syllogistic with label $t \in\{\mathrm{A}, \mathrm{E}, \mathrm{I}$, O\}, and let $h$ be a homomorphism from VENN to syllogistic s.t. $h(\delta)=$ StP . A diagrammatic configuration is $C f g(\delta)=\{h(\delta) \mid \delta \in \Delta\}$.

\section{Definition 2}

(Free ride) Let $\delta$ be a diagram for a categorical proposition in VENN and let $\Delta$ be a finite sequence of diagrams for categorical propositions in $V E N N$, we say $\delta$ is a free ride from $\Delta, \Delta-\delta$, if and only if $\delta=\Delta$ or there is a sequence of applications of rules I-VI that allows us to transform $\Delta$ into $\delta$. 
Now, provided we interpret diagrams as physical situations, we can account for the next statement:

\section{Proposition 1}

(Non-monotonicity) VENN allows non-monotonicity.

Proof. We prove this by producing a counter-example. Suppose $V E N N$ allows monotonic inference and let $C f g(\Delta)=$ MAPSAM. From such configuration we can get a free ride to the configuration $C f g(\delta)=\mathrm{SAP}$, i.e., $\Delta-\delta$, as in Figure 6 .

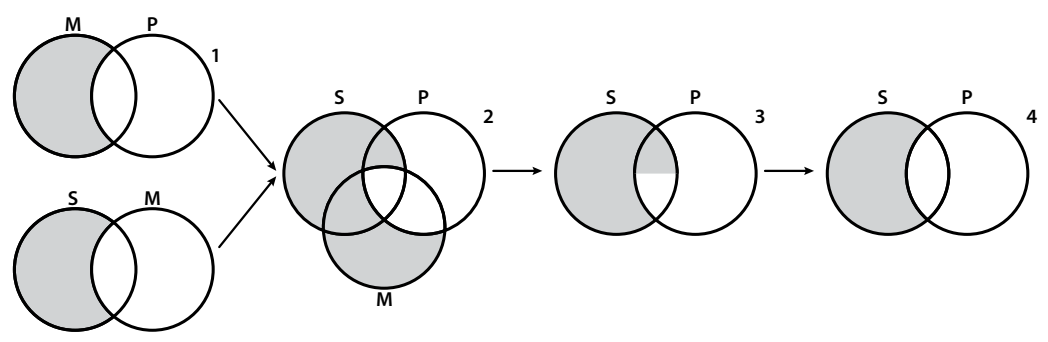

Figure 6. MAPSAM $\triangle \mathrm{SAP}$

Now, as we did in Example 2, pick a contradictory diagram $\delta^{\prime}=\neg \delta$, i.e. pick $C f g\left(\delta^{\prime}\right)=\mathrm{SOP}$, and add it by an addition rule (namely, introduction of a region). Since $\delta$ and $\delta$ ' are opposite physical situations, they cannot occupy more than one place simultaneously and no space can be occupied by the two of them, so the addition of $\delta$ ' implies the erasure of $\delta$ by an erasure rule (namely, erasure of a diagrammatic object). This avoids any application of rule V, i.e., it disallows any combination of contradictory diagrams, and hence, while $\Delta-\delta$ holds, $\Delta \cup\left\{\delta^{\prime}\right\}-\delta$ does not (Fig. 7). ${ }^{6}$

6 At this point, maybe an anecdote is in order: we came to appreciate this situation after teaching syllogistic with VENN. Usually students would enter in conflict while drawing combinations of diagrams for universal and particular statements. They normally would think that, once we draw an $\mathrm{X}$ sequence in a region, that region cannot be occupied by a shadow, or vice versa. But later they find out that, sometimes, a new shadow is needed where there is an $\mathrm{X}$ (or vice versa), and then they have to erase the $\mathrm{X}$ (or the 


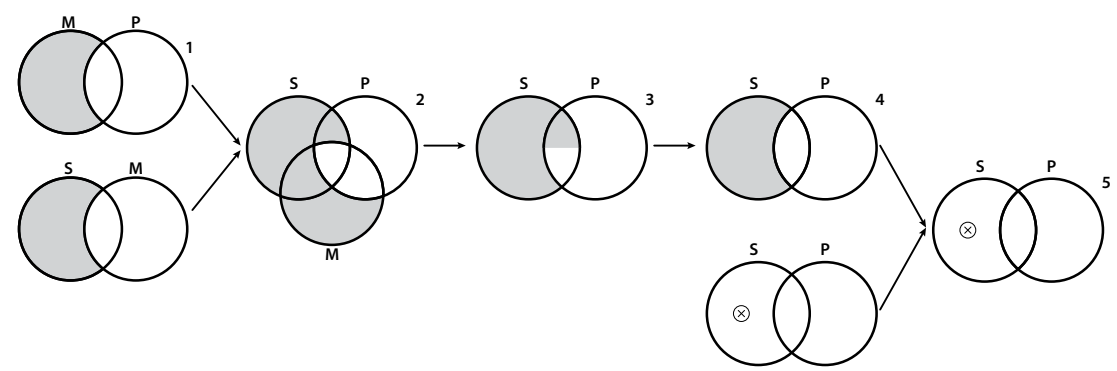

Figure 7. MAPSAMUSOP SAP does not hold.

We can extract two consequences from this result. First, that VENN provides a logical framework able to model some form of non-monotonic diagrammatic inference, provided diagrams are rightly interpreted as physical situations. ${ }^{7}$ And second, that we can advance the idea of non-monotonic diagrammatic systems that we could approximate, for example, with rules à la Nute (2003), by letting $>$ denote a preference relation over diagrams, $>$ denote a strong free ride, $\triangleright$ a weak free ride, and $\triangleleft$ and $\triangleleft$ represent not being strong and weak overdetermined alternatives:

shadow), in order to incorporate a new diagrammatic object; but in doing so, students often assume that an $\mathrm{X}$ and a shadow cannot occupy the same place simultaneously and that such space can only be occupied by one of those diagrammatic objects. What this anecdote indicates, in our opinion, is that the physical constraints of VENN are quite natural when doing diagrammatic reasoning and that they modify the a priori understanding of diagrammatic inference.

7 It seems that we can restrict the notion of a free ride solely by disallowing the combination of contradictory diagrams and blocking the inference while having premises not combined. This ingenious solution, pointed out to us by one of the referees, allows us to represent contradictory diagrams while disallowing monotonicity. We believe this solution, and the one we have advanced here, are equivalent in so far as both disallow the rule of conflicting information; however, we think that appealing to the fact that diagrams are physical situations provides an explanatory advantage in that it gives us a plausible explanation of the rejection of such rule. 
1) $\Delta-\delta$ iff
a) $\Delta=\delta$ or
b) $\exists \Delta^{\prime} \subseteq \Delta: \Delta^{\prime}-\delta$

2) $\Delta \triangleright \delta$ iff
a) $\Delta-\delta$ or
b) $\Delta \triangleleft \neg \delta$ and
i) $\exists \Delta^{\prime} \subseteq \Delta: \Delta^{\prime} \triangleright \delta$ or
ii) $\Delta>\neg \delta$

3) $\Delta<\delta$ iff
a) $\Delta \neq \delta$ and
b) $\forall \Delta^{\prime} \subseteq \Delta: \Delta^{\prime}<\delta$

4) $\Delta \triangleleft \delta$ iff
a) $\Delta<\delta$ and
b) $\Delta \neg \neg \delta$ or
a) $\forall \Delta^{\prime} \subseteq \Delta: \Delta^{\prime} \triangleleft \delta$ and
a) $\delta \ngtr \neg \delta$

Rule 1 (3) would describe classical diagrammatic systems by defining classic or strong notions of free ride ( $\delta$ is a strong free ride from $\Delta$ if and only if $\delta$ is the configuration given by $\Delta$ or if there is a subset $\Delta^{\prime}$ of $\Delta$ from which $\delta$ is a strong free ride); while rule 2 (4) would describe non-classical diagrammatic systems by defining a non-classic or weak notion of free ride ( $\delta$ is a weak free ride from $\Delta$ if and only if $\delta$ is a strong free ride from $\Delta$ or if $\neg \delta$ is not a strong overdetermined alternative and either for some subset $\Delta^{\prime}$ of $\Delta, \delta$ is a weak free ride or $\delta$ is preferred over $\neg \delta$ ).

With these rules, we can state some relations between classical and non-classical diagrammatic notions of inference. For example, one plausible but trivial relation would establish that diagrams obtained by way of strong free rides must also be obtained by way of weak free rides (but not inversely); and conversely, that weak overdetermined alternatives must imply not having strong overdetermined alternatives (but not inversely):

\section{Proposition 2}

(Subalterns) If $\Delta-\delta$, then $\Delta \triangleright \delta$; and if $\Delta \triangleleft \delta$, then $\Delta \triangleleft \delta$.

Another relation would say that strong free rides must not conflict with strong overdetermined alternatives, and the same for weak free rides and weak overdetermined alternatives: 


\section{Proposition 3}

(Contradictories) There is no $\delta$ s.t. $\Delta-\delta$ and $\Delta<\delta$, and there is no $\delta$ s.t. $\Delta \triangleright \delta$ and $\Delta \triangleleft \delta$.

Finally, a last couple of relations would establish that strong free rides and weak overdetermined alternatives must not conflict, but weak free rides and strong overdetermined alternatives may coexist:

\section{Proposition 4}

(Contraries) There is no $\delta$ s.t. $\Delta-\delta$ and $\Delta \triangleleft \delta$.

\section{Proposition 5}

(Subcontraries) For all $\delta, \Delta \triangleright \delta$ or $\Delta \triangleleft \delta$.

These propositions show the coherence of these diagrammatic relations. We can represent this coherence diagrammatically with the next square of opposition:

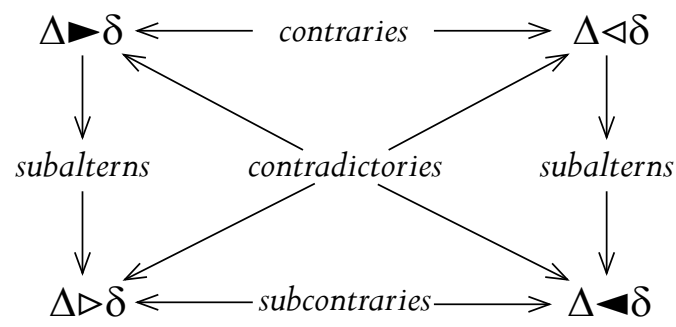

Figure 8. A square of opposition

In order to illustrate these relations between classical and nonclassical diagrammatic systems, consider a couple of examples.

\section{Example 3}

Consider an instance of a valid syllogism, say a Barbara syllogism. In Figure 9 we can see $C f g(\mathrm{SAP}) \neq C f g(\mathrm{MAPSAM})$, but for some subset of $C f g($ MAPSAM $), C f g(\mathrm{SAP})$ is indeed a strong free ride, which means that $C f g(\mathrm{SAP})$ is also a weak free ride (Proposition 2), but it is not a strong nor a weak overdetermined alternative (Propositions 3 and 4). 


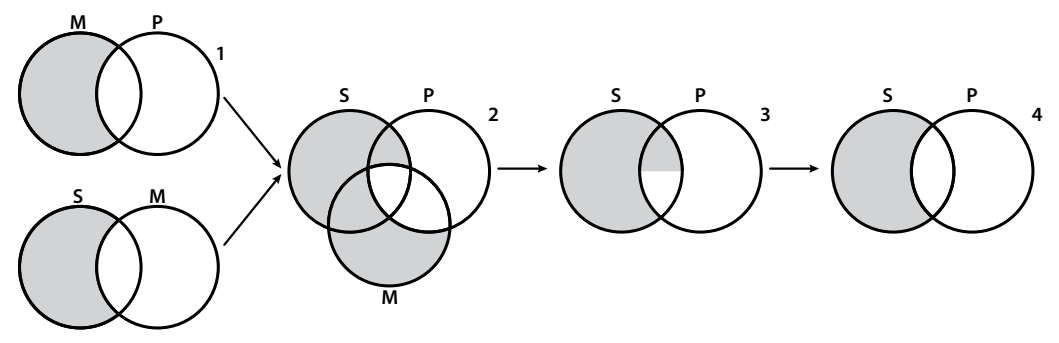

Figure 9. MAPSAM $\square$ SAP and MAPSAM $\triangle \mathrm{SAP}$

\section{Example 4}

As a second case, consider an invalid syllogistic form, say MIPSIM $\therefore$ SIP. Since this syllogistic form is invalid, it should be the case that its diagrammatic representation must be an overdetermined alternative. Indeed, in Figure 10 we can see that $C f g($ SIP $) \neq C f g($ MIPSIM) and that for all the remaining combinations or subsets of diagrams, $C f g(\mathrm{SIP})$ is not a strong free ride, which means that $C f g(\mathrm{SIP})$ is not a strong free ride but a strong overdetermined alternative (Proposition 3), from which it follows that $C f g(\mathbf{S I P})$ is admissible if regarded as a weak free ride (Proposition 5), but both, $\longrightarrow$ and $\triangleright$, do not hold (Proposition 4).

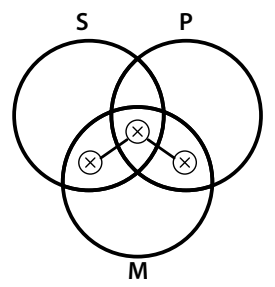

Figure 10. MIPSIM $\triangleleft$ SIP or MIPSIM $\triangleleft$ SIP

\section{Conclusions}

After a brief revision of the notion of diagrammatic reasoning, we argued that VENN provides a logical framework able to model some form of non-monotonic diagrammatic reasoning, provided diagrams are interpreted as physical situations. Then we suggested the idea 
that we could characterize classical and non-classical (i.e., nonmonotonic) systems of diagrammatic reasoning and we pointed out some possible bridges between such systems.

We think these results, although purely theoretical so far, are interesting in and of themselves, but they are also enthralling because they open the possibility of treating diagrammatic systems not only in logical but in extra logical terms, thus allowing deeper philosophical research: Is there something as a paraconsistent diagram? Is there an intuitionistic or relevantist interpretation of diagrams? Can diagrams allow (not just represent) inferences beyond deductive paradigms? We think answers to these questions deserve further research.

\section{Acknowledgements}

We would like to thank the anonymous reviewers for their precise corrections and useful comments. Financial support given by UPAEP Grant 30108-1008.

\section{References}

Allwein, G., J. Barwise. (1996). Logical Reasoning with Diagrams, Oxford University Press: New York.

Bochman, A. (2011). "Logic in nonmonotonic reasoning" En Non-monotonic reasoning. Essays celebrating its 30th anniversary. College Publications.

Lagrange, J.L., A.C. Boissonnade, V.N. Vagliente. (1997). Analytical Mechanics. Kluwer Academic: Dordrecht.

Bök, A.F. (1766). Sammlung der Schriften, welche den logischen Calcul Herrn Ploucquets betreffen. Frankfurt U.A.

Descartes, R., V.R. Miller, R.P. Miller. (1983). Principles of Philosophy. Reidel: Dordrecht.

Dieudonné, J. (1960). Foundations of modern analysis. Academic Press: New York.

Dung, P.M. (1995). "On the Acceptability of Arguments and its Fundamental Role in Nonmonotonic Reasoning, Logic Programming and n-Person Games”. En Artificial Intelligence, 77(2), 321-357. 
Englebretsen, G. (1992). Linear Diagrams for Syllogisms (with Relationals). En Notre Dame Journal of Formal Logic, 33(1), 37-69. DOI: 10.1305/ndjfl/1093636009

Moore, R.C. (1985). "Semantical Considerations on Nonmonotonic Logic". En Artifical Intelligence, 25(1): 75-94.

Gardner, M. (1958). Logic Machines and Diagrams. McGraw-Hill: New York.

Hamilton, W., H.L. Mansel, J. Veitch. (1860). Lectures on Metaphysics and Logic. Gould and Lincoln: Boston.

Hammack, R. H. (2013). Book of Proof. Virginia Commonwealth University: Richmond.

Hammer, E. (1995). "Diagrammatic Logic". En Gabbay D.M., F. Guenthner. Handbook of Philosophical Logic (Vol. 4). Elsevier North Holland: Amsterdam.

Kant, I., J.M.Young. (1992). Lectures on Logic. Cambridge University Press: Cambridge.

Karnaugh, M. (1954). "Map Method for Synthesis of Logic Circuits”. Electrical Engineering, 73(2): 136-136. DOI: 10.1109/ee.1954.6439241

Kepler, J., EJ. Aiton, A.M. Duncan, J.V. Field. (1997). The Harmony of theWorld by Johannes Kepler:Translated into English with an Introduction and Notes. Philadelphia: American Philosophical Society.

Kraus, S., D. Lehmann, M. Magidor. (1990). "Nonmonotonic Reasoning, Preferential Models and Cumulative Logics”. En Artifical Intelligence, 44, 167-207.

Lambert, J.H. (1764). Neues Organon oder Gedanken Uber die Erforschung und Bezeichnung desWahren und dessen Unterscheidung vom Irrthum und Schein. Bey Johann Wendler: Leipzig.

Larkin, J.H., H.A. Simon. (1987). "Why a Diagram is (Sometimes) Worth Ten Thousand Words". Cognitive Science, 11(1), 65-100. DOI: 10.1111/j.15516708.1987.tb00863.x

Leibniz, G. W., and Couturat, L. 1961. Opuscules - et fragments inedits de Leibniz, extraits des manuscrits de la Bibliothèque royale de Hanovre, G. Olm, Hildesheim.

Leibniz, G.W., P. Remnant, J. Bennett. (1996). New essays on human understanding. Cambridge University Press: Cambridge.

Llull, R.; P. Posa. (1501). Ars Magna. Impressum per Petru Posa: Barchne.

Londey, D.; C. Johanson. (1987). The Logic of Apuleius: Including a Complete Latin Text and English Translation of the Peri Hermeneias of Apuleius of Madaura. E.J. Brill: Leiden.

McCarthy, J. (1980). "Circumscription. A Form of Non-Monotonic Reasoning”. Artifical Intelligence 13, 27-29.

McDermott, D.J. Doyle. (1980). “Non-Monotonic Logic I”. Artificial Intelligence 13, 41-72. 
Moktefi, A., S. Shin. (2013). Visual Reasoning with Diagrams. Birkhauser: Heidleberg.

Murner, T. (1509). Logica Memorativa. Argentoratum.

Nakatsu, R. (2010). Diagrammatic Reasoning in AI. Wiley: Hoboken.

Nelsen, R.B. (1993). Proofs withoutWords: Exercises in Visual Thinking. The Mathematical Association of America: Washington.

Nute, D. (2003). “Defeasible Logic”. Bartenstein, O., U. Geske, M. Hannebauer, O. Yoshie, eds. Web Knowledge Management and Decision Support, volume 2543 of Lecture Notes in Computer Science. Springer Berlin Heidelberg, 151-169.

Pagnan, R. (2012). “A Diagrammatic Calculus of Syllogisms”. En Journal of Logic, Language and Information 21(3), 347-364. DOI: 10.1007/s10849-011-9156-7

Peirce, C.S. (1906). "Prolegomena to an Apology for Pragmaticism". En Monist, 16(4), 492-546. DOI: $10.5840 /$ monist190616436

Pollock, J. (1995). Cognitive Carpentry. Cambridge, MA: Bradford/MIT Press.

Polster, B. (2004). Q.E.D.: Beauty in Mathematical Proof. Walker \& Co: New York.

Reiter, R. (1980). “A Logic for Default Reasoning”. En Artificial Intelligence 13, 81-132.

Shimojima, A. (1996). "Operational constraints in diagrammatic reasoning". Allwein, G., J. Barwise. Logical reasoning with diagrams. Oxford University Press: New York.

Shin, S. (1994). The Logical Status of Diagrams. Cambridge University Press: Cambridge.

Shoham, Y. (1987). “A Semantical Approach to Nonmonotonic Logics”. Ginsberg, M. L., ed. Readings in Non-Monotonic Reasoning. Los Altos, CA: Morgan Kaufmann, 227-249.

Stevin, S. (1586). De beghinselen der weegh const. Leyden.

Tennant, N. (1986). "The Withering Away of Formal Semantics?". En Mind \& Language 1(4), 302-318. DOI: 10.1111/j.1468-0017.1986.tb00328.x

Venn, J. (1880). "On the Diagrammatic and Mechanical Representation of Propositions and Reasonings". En Philosophical Magazine Series 5, 10(59), 1-18. DOI:10.1080/14786448008626877 